\title{
The potential role of elastography in differentiating between endometrial polyps and submucosal fibroids: a preliminary study
}

\author{
Sławomir Woźniak \\ $3^{\text {rd }}$ Chair and Department of Gynecology, Medical University of Lublin, Lublin, Poland
}

\begin{abstract}
Endometrial polyps and submucosal fibroids are common causes of abnormal uterine bleeding (AUB) and less commonly infertility. The prevalence of such intrauterine lesions increases with age during the reproductive years, and usually decreases after menopause. The first-line imaging examination in the diagnosis of endometrial polyps as well as submucosal fibroidsis ultrasound, but its accuracy is not obvious. Elastography is an ultrasound-based imaging modality that is used to assess the stiffness of examined tissues. Considering the fact that endometrial polyps derive from soft endometrial tissue and submucosal fibroids are made of hard muscle tissue, elastography seems a perfect tool to differentiate between such lesions. I present two groups of patients with AUB and intrauterine lesions suspected on ultrasound. In the first group of patients, elastography showed that the stiffness of the lesion was similar to the endometrium and softer than the myometrium. During hysteroscopies endometrial polyps were removed. In the second group of patients, elastography showed that the stiffness of the lesion was similar to the myometrium and harder than the endometrium. During hysteroscopies submucosal fibroids were removed. In both groups, the diagnosis was confirmed by the pathological examination in all cases. It was demonstrated that with the use of elastography it is possible to assess the stiffness of intrauterine lesions, which may be useful in differentiating between endometrial polyps and submucosal fibroids.
\end{abstract}

Key words: elastography, ultrasound, endometrial polyps, submucosal fibroids.

\section{Introduction}

Endometrial polyps and submucosal fibroids are common causes of abnormal uterine bleeding (AUB) and less commonly infertility. The prevalence of such intrauterine lesions increases with age during the reproductive years, and usually decreases after menopause. The first-line imaging examination in the diagnosis of endometrial polyps as well as submucosal fibroidsis ultrasound, but its accuracy is not obvious.

Elastography is an ultrasound-based imaging modality that is used to assess the stiffness of examined tissues. Considering the fact that endometrial polyps derive from soft endometrial tissue and submucosal fibroids are made of hard muscle tissue, elastography seems a perfect tool to differentiate between such lesions.

\section{Methods and Results}

In this study, initially cases of two patients with AUB are described. The first patient (39 years old, two vaginal deliveries in history, generally healthy) reported heavy menstrual periods and mid-cycle spotting for the last 9 months. The gynecological examination did not reveal any pathology. Transvaginal ultrasound (Samsung V-20) showed a hyperechogenic intracavitary uterine lesion (5 $\mathrm{mm} \times 4 \mathrm{~mm}$ ) (Fig. 1). The second patient (41 years old, one vaginal delivery in history, generally healthy) reported heavy menstrual periods with blood clots for the last 12 months. The gynecological examination did not reveal any pathology. Transvaginal ultrasound showed an intracavitary uterine lesion $(9 \mathrm{~mm} \times 5 \mathrm{~mm}$ ) of irregular echogenicity (Fig. 2). In both patients the elastography option was then used (Elastoscan ${ }^{\circledR}$ ). Both patients signed informed consent to use elastography and prolong the examination, the study was accepted by a local bioethics committee (KE-0254/120/2012). In elastography the applied force (either active or passive, i.e. due to respiratory or cardiovascular pulsation) induces a strain in examined tissues: higher in soft tissues and lower in hard ones. Strain ratios are visualized on a color map creating an elastogram showing the stiffness of examined areas. During the examination patients were breathing normally and the operator did not apply pressure to the cervix - the elastographic 

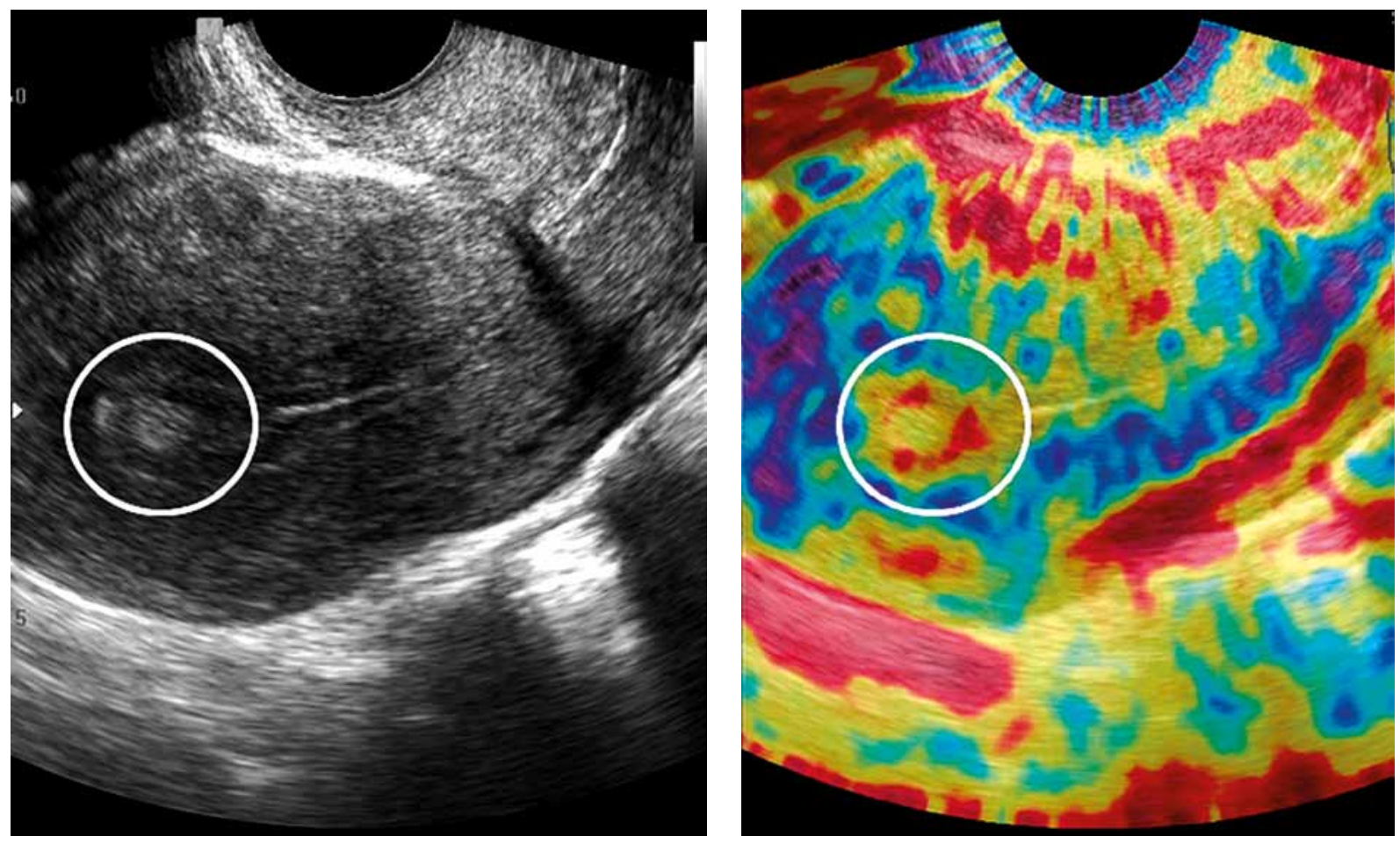

Fig. 1. An intrauterine lesion (left circle) was observed on grayscale ultrasound. Elastography showed that the stiffness of the lesion was similar to the endometrium and softer than the myometrium (right circle). The pathological examination confirmed the diagnosis of an endometrial polyp
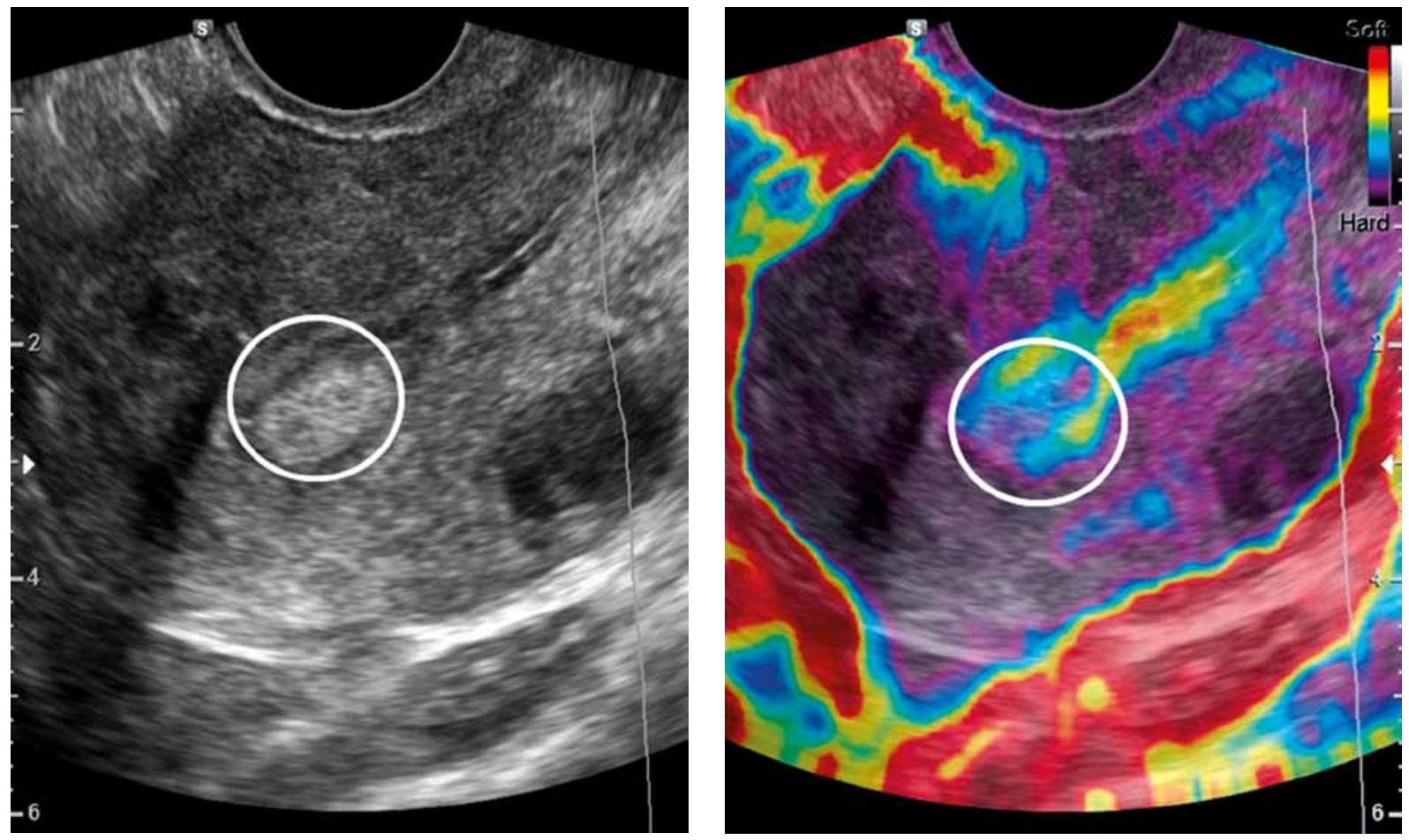

Fig. 2. An intrauterine lesion (left circle) was observed on grayscale ultrasound. Elastography showed that the stiffness of the lesion was similar to the myometrium and harder than the endometrium (right circle). The pathological examination confirmed the diagnosis of a submucosal fibroid 
image of the uterus was generated by patient's breathing movements and arterial pulsation. Elasticity of the regions of interest was assessed using a color map as red (soft), yellow (medium soft), blue (medium hard) and dark purple (hard). An $\alpha$-blend mode was used, where the elastographic color map was blended with the grayscale image. The enhancement was adjusted to visualize hard myometrium and soft endometrium around the intrauterine lesion. In the first patient, the lesion's stiffness was softer than the myometrium and similar to the endometrium (Fig. 1). In the second patient, the lesion's stiffness was harder than the endometrium and similar to the myometrium (Fig. 2). Finally, in both patients, a hysteroscopy was performed: in the first patient, an endometrial polyp was found and removed. In the second patient, a submucosal fibroid was found and removed. In both cases the diagnosis was confirmed by the pathological examination. The postoperative course was uncomplicated in all cases.

In the second stage of the study, additional 14 patients with AUB due to endometrial polyps or submucous fibroids were recruited. The clinical characteristics of the studied 16 patients are shown in Table I. Elastographic evaluation of the intrauterine lesions was made according to the above-described method. Statistical analysis was performed using Statistica software (version 10, Statsoft, Tulsa, OK, USA). The sensitivity, specificity, negative predictive value (NPV), positive predictive value (PPV) for elastography in detecting submucosal fibroids were $87.5 \%$ and $75 \%$, respectively and in cases of endometrial polyps $-85.7 \%$ and $77.7 \%$, respectively.

\section{Discussion}

It was demonstrated that with the use of elastography it is possible to assess the stiffness of intrauterine lesions, which may be useful in differentiating between endometrial polyps and submucosal fibroids. To the best of the author's knowledge, this is the first report addressing this issue. Differentiating between endometrial polyps and submucosal fibroids may be of clinical importance. Expectant management may be implemented in selected cases of endometrial polyps [1].
The availability of hysteroscopic morcellators for submucosal fibroids should also be considered [2].

According to current guidelines, transvaginal ultrasound should be the first-line imaging modality in premenopausal patients with abnormal uterine bleeding. Saline infusion sonohysterography and diagnostic hysteroscopy should be used in differential diagnosis and characterization of intrauterine lesions [1]. However, sonohysterography is more expensive and time consuming than classic transvaginal ultrasound (including elastography), requires the use of catheters and is an invasive procedure (even though minimally), thus carries the risk of complications (pain, infection etc.). Moreover, during sonohysterography pedunculated fibroids may mimic polyps while broad-based polyps may appear as fibroidlike ones [3]. These drawbacks justify the search for new diagnostic approaches distinguishing intrauterine lesions. For instance, power Doppler vascular patterns were proved to be useful in differentiating between endometrial polyps and submucosal fibroids [3].

Naturally, at this stage it is far too early to discuss the possible clinical value of elastographic assessment of intrauterine lesions, hence larger studies addressing this issue need to be performed first. The International Endometrial Tumor Analysis (IETA) group attempted to unify the terms and definitions to describe ultrasound findings in the uterine cavity and to develop recommendations for a standardized measurement technique for both endometrial thickness and intracavitary lesions [4]. The aim of the IETA consensus was to form the basis for prospective studies to predict the risk of different endometrial pathologies based on their ultrasound appearance [4]. Taking into consideration our initial experience with elastography and the previously shown usefulness of elastography in differentiating between endometrial pathologies [5], it seems reasonable to postulate that elastographic assessment of endometrial lesions should also be included in future research protocols.

\section{Conclusions}

It is possible to assess the stiffness of intrauterine lesions with the use of elastography, which may be use-

Tab. I. Characteristics of the studied population

\begin{tabular}{lll}
\hline Age (years; median, range) & & $46 ; 41-54$ \\
\hline \multirow{2}{*}{ Menopausal status } & Premenopausal $(n, \%)$ & 12 \\
\cline { 2 - 3 } & Postmenopausal $(n, \%)$ & 4 \\
\hline \multirow{2}{*}{ Symptoms } & Spotting $(n, \%)$ & 6 \\
\cline { 2 - 3 } & Heavy menstrual bleeding $(n, \%)$ & 10 \\
\hline Endometrial polyp $(n, \%)$ & & 7 \\
\hline Submucosal fibroid $(n, \%)$ & & 9 \\
\hline
\end{tabular}


ful in differentiating between endometrial polyps and submucosal fibroids.

Although promising the results of this preliminary study need to be confirmed in a larger survey including intrauterine lesions of different sizes and histopathological character.

\section{Disclosure}

Authors report no conflict of interest.

\section{References}

1. Singh S, Best C, Dunn S, et al. Abnormal uterine bleeding in pre-menopausal women. J Obstet Gynaecol Can 2013; 35: S1-28.

2. Pakrashi T. New hysteroscopic techniques for submucosal uterine fibroids. Curr Opin Obstet Gynecol 2014; 26: 308-313.

3. Cil AP, Tulunay G, Kose MF, Haberal A. Power Doppler properties of endometrial polyps and submucosal fibroids: a preliminary observational study in women with known intracavitary lesions. Ultrasound Obstet Gynecol 2010; 35: 233-237.

4. Leone FP, Timmerman D, Bourne T, et al. Terms, definitions and measurements to describe the sonographic features of the endometrium and intrauterine lesions: a consensus opinion from the International Endometrial Tumor Analysis (IETA) group. Ultrasound Obstet Gynecol 2010; 35: 103-112.

5. Preis K, Zielinska K, Swiatkowska-Freund M, et al. The role of elastography in the differential diagnosis of endometrial pathologies - preliminary report. Ginekol Pol 2011; 82: 494-497. 